\title{
Change Processes: Multi-Disciplinary Teamwork
}

\begin{abstract}
This chapter examines the importance of multi-disciplinary team-working to the management of antimicrobial stewardship and behaviour change processes. MDT has played a major role in improving communication and reducing professional boundaries that had previously contributed to high levels of infection; extended use of, often inappropriate antibiotics and poor patient outcomes. The functioning of such teams facilitates the role of clinical pharmacy.
\end{abstract}

Keywords Multi-disciplinary team working - Behaviour change · Huddling

There is a meeting of minds of all the different people so the future for the work is quite bright. (Hospital Administrator)

The main thing is the excellent team working you have done here. (Intern Doctor)

One of the anticipated outcomes of the project from the funders' perspective was 'improved knowledge and practice'. Knowledge comes in many forms and it is often associated too narrowly with forms of explicit scientific or clinical knowledge. The interpretation of laboratory results is an example of this kind of knowledge. Changing behaviour requires more

(C) The Author(s) 2020

L. Ackers et al., Anti-Microbial Resistance in Global Perspective, https://doi.org/10.1007/978-3-030-62662-4_7 
than an increase in this form of knowledge. Explicit knowledge works hand-in-hand with forms of tacit knowledge and it is these knowledge combinations that stimulate change. Our research on the gains to NHS staff from periods of volunteering in Uganda (Ackers et al. 2017) has emphasised the relative importance of tacit knowledge; of experience in leadership, cultural understanding, communication, and team working. Team-working, as much as any gain in scientific or clinical know-how is the connecting knowledge that has made this project a success. Indeed, it is the positive experience of team-working that has driven the quest for scientific knowledge (to know more). This fits with our conceptualisation of behaviour change; it is not new knowledge that drives motivation; but motivation that drives the thirst to learn. It is interesting to reflect here on the assumed knowledge gains to NHS volunteers expressed in the funding call. The emphasis on these more tacit forms of knowledge and leadership skills for NHS staff is very evident whilst the knowledge gains to LMIC staff appear to be focused on more explicit skills (prescribing and IPC). Somekh makes an interesting point about the relationship between 'training' and education or knowledge suggesting that the concept of knowledge has a more sophisticated tone whilst 'training has strong cultural associations with the teaching of low-level skills' (2006: 19). There may be other ways of understanding this relationship perhaps seeing training as more linked to technical know-how and an instruction on what to do in certain circumstances rather than a more holistic understanding of the knowledge underlying that.

It is resoundingly clear from the interviews, attendance at meetings and from on-going observational work on the ground that the key success in this intervention hinges on the achievement of genuine, multi-disciplinary team-working with each cadre showing mutual respect and willingness to learn from each other. This level of team-working did not exist prior to the project and many of the key actors had weak relationships with each other. They may have known about the existence of the laboratory, for example, but not developed a relationship with the staff there or understood the kind of contribution the laboratory could make to their work and their patients. Similarly, they may know that there are pharmacists at the hospital but did not understand their roles and knowledge and interact with them in any meaningful way, particularly in clinical decisionmaking. There was certainly minimal engagement with the nursing and midwifery team who are the most present on the ward and have most direct patient engagement. 
The 'magic' that fostered this team working (as one respondent referred to it) is complex. Many respondents attributed it directly to the project. However, important structural changes took place in the hospital at around the same time. A new in-charge had been appointed on the ward, for example. The (externally funded) laboratory had begun to function at a much higher level. The decision to appoint an inventory manager in pharmacy stores also released a senior pharmacist with a passion for clinical pharmacy enabling him to engage directly with the team. This is an excellent example of effective task-shifting. From a project perspective, the placement of $\mathrm{K} 4 \mathrm{C}$ staff and professional volunteers on the ward on a continuous basis undoubtedly stimulated team-working and specifically the empowerment of the nursing and midwifery team. The involvement of a senior obstetrician volunteer also stimulated the setting up of daily ward meetings which staff reported to have had an impact of team working.

The decision to improve the ward infrastructure and basic infection control; decorate; provide trolleys to enable the nursing and midwifery staff to work effectively; and the provision of instruments in the evacuation room have made staff feel valued, improving motivation and job satisfaction enabling them to use the skills they have more effectively. All of these factors are of great importance. However, we must not underestimate the impact of the introduction and embedding of culture and sensitivity testing to the team-building process. The culture and sensitivity testing process is inherently a team-based activity; it engages all staff irrespective of cadres. Having the laboratory results provides an 'objective' focus for interest to coalesce around; it stimulates team discussions and active pharmacy engagement and creates the environment for genuinely patient-centred care. Having the evidence-base for rational prescribing undermines any artificial disciplinary hierarchies (who knows best). One respondent refers to the impact of having laboratory results on hierarchies:

Sometimes there can be ego - that the doctor or pharmacist thinks, 'I am the overall boss so I can't be directed on what to do' but with the data that goes down. 
Laboratory results provide a critical focal point for what some pharmacy teams in the UK refer to as 'huddling'. ' Gardner et al. characterise huddling as a mechanism to 'support inter-professional communication and collaborative practice' (2018: 16). The consequences of rational prescribing as a central component of patient care have delivered tangible and often quite surprising results. Staff all remember and refer directly to cases where women's lives have been saved and women have got better remarkably quickly as a result of this process. The sheer tangibility of these outcomes has had a major motivational effect on all concerned; so, teamworking becomes enjoyable and immediately rewarding. We cited the words of a Ugandan health worker in our previous book to illustrate the devastating impact on health worker motivation of having 'people dying in your hands [when] you know what to do but don't have the things to help them' (Ackers and Ackers-Johnson 2016: 96). Seeing patients survive and leave hospital has a huge motivational impact and drives a thirst for knowledge. Some of the potential rewards to working on the PNG wards relates to the nature of the work itself. Many respondents spoke of the negative aspects of the work before the project in terms of the offensive smelling wounds. Once this problem was resolved and women were seen to be getting better quicker, health workers noted the rewards of working in this area:

The [nurses and midwives] are proud; they really feel good when they say they don't have any septic patient on the ward which means they are trying to prevent sepsis.

When we asked a midwife if she had had experience of this kind of team working in other areas, she replied that team working did coalesce around obstetric emergencies on labour ward. But she made the interesting point that these emergencies are short-lived events and the fact that women with sepsis stay in the hospital for longer helps to build these relationships with patients and a more vested interest in, and responsibility for, their well-being:

[Have you seen that teamwork, motivation, and collective interest in the patient in any other place you have worked?]

\footnotetext{
${ }^{1}$ http://www.pulsetoday.co.uk/pulse-intelligence-your-practice/regulation/how-ourmorning-huddles-improved-practice-teamworking/20037047.article.
} 
In labour suite when there is an emergency there is teamwork but once the emergency is over, they move on. But here the patients stay longer so we see the same patient. We want to know how they are doing - if they are getting better so there is continuity of care here.

A knock-on consequence of this has been the fact that the ward is no longer 'the laughingstock' of the hospital; and is seen rather as a centre of excellence to be envied. This has been openly acknowledged in hospital meetings as having a massively motivational impact on all staff concerned. The in-charge describes this change:

Things have totally changed. Before the project, the ward faced challenges. The hospital assesses every unit regularly along various indicators. Before, the unit always came out badly and we were always hearing our name in meetings as a result; but now things are much improved. The hospital director said to me; 'What's happening on post-natal? You people are quiet - the deaths are not there as they used to be'.

The Chair of IPC Committee echoes this view:

Sincerely the trend has changed. Maternity was the ward we laughed at and now it is the ward we all envy.

When a team is successful, and its success is acknowledged, new members seek to join the team and gain that experience. And as they leave the ward, they are motivated to take that experience to their new ward or facility. The following section explores some of the team working relationships in more detail and identifies areas for future improvement.

\section{Perceptions of Team Working}

Every respondent reported the improvement in teamwork and identified this as the source of change on the wards.

We are now working hand-in-hand with the pharmacists, the laboratory, and the doctors'. (In-charge)

I saw a gap with the midwives and doctors. You pass by on a ward round they don't always tell you what challenges they face. I found a burst abdomen and the pus was a lot, so I found myself cleaning the wound. 
On ward rounds you don't usually see the wounds so you can't know the problem. Now I combine the ward round with wound dressing (it can take me up to $10 \mathrm{pm})$. We do the wound dressing together with the midwives, so we work together. (Intern Doctor)

One midwife describes the team-working that has developed. She particularly emphasises the engagement of doctors which she puts down, in part, to the sheer tenacity of the $\mathrm{K} 4 \mathrm{C}$ midwives which they have now 'inherited':

We are working hand-in-hand together as a team. Before the doctor was not there but nowadays doctors are responding. (The change) is because of the teamwork those people introduced. They showed us good leadership. They would follow-up and afterwards they call the doctor and he comes even if they first deny we call again even the senior doctors. They come - it is a big change.

The reference here to senior doctors is interesting. The general view reinforced by observations on the ward and the experience of a strike by intern doctors, in part due to lack of supervision (in September 2019), is that interns are largely left to cope and the presence of senior doctors is at best sporadic. The relatively junior status of the intern doctors may, to some extent, explain their willingness to engage in teamworking, benefit from mentoring by the pharmacist and their appetite for learning. However, concern was also expressed about intern 'burnout' in the absence of supervision and the challenges of continuity when interns rotate every 2 months. The professional volunteers play an important role in supporting interns in the absence of senior Ugandan doctors. The incharge nurse took on a major role in orienting new interns on the ward which will help them to integrate in the team but this puts an additional burden on the in-charge and could and should be fulfilled by more senior doctor engagement. It was clear from the discussion in one of the IPC Committees that the tension between doctors persists in other areas of the hospital and was previously evident on the PNG ward:

Sometimes you find a pharmacist has changed a prescription and then on the ward round the doctor changes it back to a drug the patient is resistant to (Midwife on IPC Committee)

A pharmacy intern reflects on this situation commenting: 
I think it should be teamwork here and respect for each other. If we advise and then the prescription is changed the clinicians come and undermine that decision without listening to the pharmacist. We can see that postnatal is taking the lead in consulting with pharmacy, but other units are relying on empirical usage designed for health centres and not for hospitals. If you are rigid on the usage you will not use the pharmacists/laboratory's advice.

Another midwife is appreciative of the team-working environment that has developed. She refers to the presence of pharmacy on the ward and the lengths the pharmacists have also gone to, to try to secure appropriate antibiotics:

It has greatly improved because right now we have pharmacists who come on a daily basis or if not, every day we can't go 3 days without seeing one who can guide us on the mothers and which drugs to take. They interact with the doctors; if you don't interact there is that collision. Right now, there is no tension. They say, 'what if we do this and there is a discussion.' We never used to have any pharmacists coming on the wards, so it was majorly the doctors dealing with the prescriptions. We have managed to reduce the irrational use of antibiotics.

The impact of medicine on multi-disciplinary team working is well established internationally. Atwal and Caldwell (2006) identified three barriers to effective team working in acute care (in the UK): these included, the 'dominance of medical power that influence interaction in teams' ((2006): 359). In a more recent editorial with specific reference to models of antimicrobial resistance in South Africa, Brink et al. consider whether models of antimicrobial stewardship developed in high-income countries translate effectively to a South African public health care context. They underline the lack of understanding of the contextual and behavioural determinants that influence local prescribing practices and present alternative models using non-specialist pharmacists, nurses and even community health workers in key stewardship roles. As doctors themselves they pose the question; 'Will doctors, the actual prescribers, and a group notoriously keen on safeguarding clinicians prescribing autonomy, embrace pharmacists and nurses as equals in the drive towards optimising antibiotic use?' (2016: 947). 
The following midwife makes a similar observation and illustrates the point above about the motivational effects of seeing a patient's condition turned around:

Now [the pharmacist] comes in daily and helps us and discusses cases with us. We had a discussion with him on Friday. He comes and looks at the files and the wound; the drugs; the length of days the patient has taken and then he gives us some alternatives. He looks at the laboratory results. There was a time he prescribed a very expensive drug and the healing was very fast. We nearly lost that young girl of 16 . The baby died. They took her to theatre twice as her uterus was necrotic, but the moment we brought that drug she improved. She has her life.

An intern doctor describes how useful he has found the specific expertise of the pharmacy team on the ward:

It's changed a lot now; the senior pharmacist comes regularly, and you may find there are 2 microorganisms sensitive to different antibiotics. Now I don't have the time to walk to the pharmacy and those people have studied medicines. I have textbook knowledge; if someone has a UTI (Urinary tract infection) I give $\mathrm{x}$. I did study this but as time goes on you get used to giving certain medicines quite often and you are not so equipped to understand how one medicine interacts with another one or if a patient has TB or is HIV positive or how to combine drugs - so a pharmacist being available on the ward has really brought great improvement.

Evidence suggests that hierarchical structures inhibit junior staff from challenging their seniors, leaving them to prescribe in a way to suit conventional senior preference rather than offering evidence-based treatment options (Broom et al. 2014). The MSI has demonstrated that effective team working challenges professional hierarchies and empowers lower cadres of staff. When team working is based on collegiality, recognition of respective skills and the role they can play in helping (especially doctors) to achieve their goals efficiently can drive change. Another senior doctor (in the IPC Committee) referred to the pharmacists as a 'tie-breakers'. 


\section{'We Are a Bridge': The Changing Role of Pharmacy IN FPRRH}

The intern pharmacist who later came to be employed on the project describes his experience of the role of pharmacy and how that has changed since the project commenced:

Interns shadow the pharmacists and I shadowed (senior pharmacist) to gain experience under supervision. Unfortunately, the pharmacists' role (in an $\mathrm{RRH}$ ) is limited to the supply chain. One of the challenges in a hospital job description - we know we can do a lot but when you get employment in a hospital there isn't scope for clinical pharmacy. We try as much as possible to show what we can do on a clinical side and we want to specialise. You need to specialise if you want your work to be noticed; at present people just see us moving around; you are everywhere, and your work isn't noticed. There should be different roles for pharmacists within the hospital and scope for clinical pharmacy. On the post-natal ward, for example, there are $6+$ doctors and 1 pharmacist (shared with the rest of the hospital). It's not effective.

[But that means employing more pharmacists?]

We - and the Pharmaceutical Society of Uganda (PSU) are advocating for specialisation. At present we manage the entire supply chain from planning, procurement, monitoring use, distribution, some physical inspection (are they keeping medicines in the right condition) and antibiotic use. We are supposed to review prescribing in the in-patient pharmacy; was it given for the right condition? And we should go through the notes, but we don't really have time to do 40 files at once. We can't review so we are just forced to dispense. So, you end up dispensing which is one of the key challenges. We want to do more, but you are really limited. It's frustrating for my future career - there is no direct career path in Uganda. The PSU came up with a draft career plan for pharmacists to discuss with the Ministry of Health. The government has been giving scholarships for clinical pharmacy but there are no positions for such people in public or private hospitals. So, why do a masters if I won't get employment anyway?

This concern about career paths is echoed in many other professions in LMICs. Creating new opportunities for higher-level qualifications and professionalisation-often encouraged by foreign experts seeking to emulate developments in their own systems (the benefits of which are often contested) - may have serious unintended consequences when higher level and remunerated positions do not evolve in parallel. This 
has been the case with degree-level programmes in nursing, midwifery and biomedical engineering, for example. There are clear benefits to higher-level capacity-building programmes when appropriate positions are available. Failing to provide such positions compounds frustration and is highly demotivating as graduates return to the same badly paid positions. There are other consequences too as graduates grasp the opportunity to avoid clinical work and seek out work with foreign NGOs. Expanding clinical pharmacy in Uganda requires both higher-level qualifications; the development of appropriate positions AND a significant increase in the volume of pharmacists in the hospitals to release them from supply chain management roles.

He then talks about his own experience of working on the project and the mentoring he has received from the pharmacist:

I'd like to do clinical pharmacy. The pharmacist has lots of experience and he is biased towards the clinical side. He is a good mentor, but he has the same frustration. He has made me appreciate the value of pharmacists in providing clinical services.

The project has changed my role a lot. There is an opportunity to really delve into what AMR is. We had heard of IPC, but we had not participated in any. I studied the theory of it at university, but this has made me learn a lot about culture and sensitivity testing and how to interpret the results. There is a key role for us (pharmacists) to interpret and guide clinicians - I've really learnt a lot! At university it was all theoretical. Now we are learning about culture and sensitivity testing and how to interpret the laboratory results. (The pharmacist) was able to show me some of things so that is an area I gained a lot. This is the role of the pharmacist to interpret the results and guide selection of antibiotics.

Pharmacy were not really doing that role before. Their job description was limited only to supply chain management. This has changed and people are now very interested - and this is because of our project. We wanted to do these things, but the project created the opportunity for us to get involved. We only became involved because of the project; the clinicians were not so keen before to involve the pharmacists - it wasn't conflict as such, but they didn't appreciate the role that pharmacy brings. At school we study together but there are no tensions as such now (with the intern doctors) now they know what pharmacists can do.

The clashes come when you try to perform their roles. For us we don't do diagnosis. You should each stick to your sides, but they recognise on the issues of antibiotic selection, they need our guidance. We are a bridge and we also know what is available in the stores and they appreciate that. 
We have information about if antibiotics are available and where we can get them from. They really appreciate it, especially the younger doctors.

His ability to extend himself and engage in clinical pharmacy reflects his supernumerary status. When we decided to take steps to retain this pharmacist at the end of his internship and support his salary through the project, we developed a clear role description so that he did not just become another pair of hands or substitute labour. This created a window of opportunity to focus on often neglected aspects of the pharmacy role and to demonstrate the value and cost-effectiveness of such roles in a regional referral hospital setting. He describes the importance of relationships and rapport to team-working:

As for the laboratory, well I know [Lab scientists] now. It is more about getting to know people who work there and building a rapport. Beforehand our only previous relationship was to requisition consumables from the stores for them but now our relationships with the nurses and the laboratory - because nurses take and collect the samples so there is much more rapport. The midwives have really appreciated the project - there is lots of change - they now attend changeover meetings of interns (when the interns rotate). [They] have stressed the need for interaction, so the nurses relate to what their colleagues are doing.

Asked to describe what his role now involves on the ward the $\mathrm{K} 4 \mathrm{C}$ pharmacist explains how he and the hospital pharmacist engage on the ward:

[The pharmacist] has a passion for clinical pharmacy - he prefers to interact with patients. Until this project there was no other opportunity for him to engage in this way. He identifies sepsis cases where pharmacy can have a real input and is working especially with the intern doctors. This was a chance for him, and he is actively involved. We don't see all the cases. We try to pick cases where we feel pharmacists can have an input and that way, we don't clash so much with the doctors. We work within our mandates so there are no clashes. Intern doctors are also using this as a chance to learn about AMR.

The hospital pharmacist echoes these concerns about hospital pharmacy in Regional Referral Hospitals. He had previously worked in the national referral hospital in Kampala and had a more clinical role in that more 
specialised context. He referred to some resistance, in the past, from doctors to clinical pharmacy:

In the past people didn't look at pharmacists as people involved in patient care. Doctors previously felt that undermined their authority. Some even hid the medical files from pharmacists. They tried to make us complete separate forms, so we were not working in the patient files. ${ }^{2}$

Asked to describe his role in FPRRH, he explains how his first year in the hospital was focused on supply chain management:

I now come in for septic and complex cases. I go on the ward and look at the entire patient file. I look at the notes, the plan of action, see if they have culture and sensitivity testing. I even go and see the patient and look at the timeline since their admission. I started doing that when I was moved from the Stores in October 2019. I worked there for a full year which was a good way to get to know the hospital but then they recruited an inventory manager. Before he came, I was tasked with management of stores and this enabled me to leave the stores and get more involved on the wards. The new role is very challenging and very rewarding. You don't go there because you have all the answers. I have had to keep reading. I know what information and online sources to look for and where to look so we can focus on a specific case and I know how to make that information relevant to a specific case but in the process I also had to teach people; nurses and midwives. It has been a continuous conversation. The patient belongs not just to me but to all of us so we have to engage in a conversation about the patient and what we can do about it. I have found this work very motivating. I spend more time on the wards now than before and every time they have a case, they want me to look at as a specialist there is now more time on the wards for me. It has been very encouraging for me and the doctors are appreciating my presence too. In the past traditionally most resistance to change came from the doctors but they are more collegial now. They have attached a certain respect to my presence because we do this in a collegial manner. The different disciplines are coming together for a common objective with mutual respect and trust. I don't come here to criticise or undermine. In fact, when I write in the notes, I write it not as a prescription but as a recommendation. They

${ }^{2}$ It was interesting to observe during a visit with this Ugandan pharmacist during a fellowship in the UK we were shown how pharmacists used a different coloured ink to make their notes on patient files. 
could disregard it. Then one doctor said, 'when he comes, and we follow his advice things turn out very well.' People's perception of pharmacy has changed tremendously. They said to me, 'we have never seen a pharmacist do what you do - how do you know to do that' and I explained to them that clinical pharmacy is part of our training. For her it was a shock; the perception was that pharmacists handle the supply chain and dispenses. I am sure the Director has had her eyes opened to what pharmacists can achieve. I think she had the same perception of every other person that pharmacists only manage procurement distribution and dispensing but now she has seen what 1 pharmacist can achieve on a very sensitive ward. The maternal mortality rate was very high and not one death has taken place since the project started. This is in line with the Sustainable Development Goals. They noted that since you guys started coming as part of our project you haven't had a maternal death from sepsis, and it used to be quite common.

Some concern was expressed at the IPC meeting, perhaps reflecting practice on other wards and not PNG that doctors were continuing to ignore the advice of the pharmacy team:

The problem was that some doctors changed the [pharmacy] recommendations and then the patient's condition did not improve so [the nurses] complained to the director in a meeting and she was very appreciative of the progress we have made.

A senior doctor challenged this attitude:

At the end of the day all our efforts should focus on the patient. The best outcome regardless of your cadre. If everyone plays his or her own role it will really be good teamwork. Without that we can't do magic here so there needs to be comprehensive teamwork and leadership. But there are issues for the administration and supplies and IPC cuts across this. Can we have more IPC supplies and more leadership involvement?

The respondent raises an important point here echoing the behaviour change literature. The development of team-working cannot happen in a vacuum and if the outcomes of that team-working, in terms of wound care or prescribing decisions cannot be operationalised due to lack of supplies then team-working will become frustrating, demotivating and collapse. 
Team-working lies at the heart of the behaviour change that we have witnessed on the post-natal and gynae wards. Recognition of the contribution of colleagues from different disciplines-and at lower pay grades - has generated 'huddles' of activity that have proved highly motivational and empowering and driven a quest for more knowledge. It is important to point out that this change has happened incredibly quickly and with relatively little investment and has proved highly cost-effective. Sam Opio notes the benefits during a follow-up visit:

There is great progress. It is clear that the departments outside of maternity are aware of the work. There is great teamwork. I was aware last time I came that people were working in silos, the laboratory, pharmacy, and the nursing team. Everything was in silos. I've seen significant teamworking. The pharmacy profile is much bigger; there is more engagement with the laboratory and the nursing team, and it is important to build on that. After documenting and disseminating we need to institutionalise and move out of silos.

Chapter 8 moves on to consider the knowledge generation and mobilisation processes that multi-disciplinary team working has enabled within the MSI.

\section{REFERENCES}

Ackers, H. L., \& Ackers-Johnson, J. (2016). Mobile professional voluntarism and international development: Killing me softly? Palgrave PIVOT. http://link.spr inger.com/book/10.1057\%2F978-1-137-55833-6.

Ackers, H. L., Ackers-Johnson, J., Tyler, N., \& A. Chatwin, J. (2017). Healthcare, frugal innovation, and professional voluntarism: A cost-benefit analysis. Cham: Palgrave PIVOT.

Atwal, A., \& Caldwell, K. (2006). Nurse's perceptions of multidisciplinary teamwork in acute health care. International Journal of Nursing Practice, 12(6), 359-365.

Brink, A. J., van de Bergh, D., Mendelson, M., \& Richards, G. A. (2016). Passing the baton to pharmacists and nurses: New models of antibiotic stewardship for South Africa. South African Medical Journal, 106(10), 947-948.

Broom, A., Broom, J., \& Kirby, E. (2014). Cultures of resistance? A Bourdieusian analysis of doctors' antibiotic prescribing. Social Science and Medicine, 110, 81-88. 
Gardner, A. L., Shunk, R., Dulay, M., Strewler, A., \& O’Brien, B. (2018, September). Huddling for high performance teams. Federal Practitioner, 16-22 https://www.ncbi.nlm.nih.gov/pmc/articles/PMC6366795/.

Somekh, B. (2006). Action research: A methodology for change and development. Maidenhead: Open University Press.

Open Access This chapter is licensed under the terms of the Creative Commons Attribution 4.0 International License (http://creativecommons.org/licenses/ by $/ 4.0 /)$, which permits use, sharing, adaptation, distribution and reproduction in any medium or format, as long as you give appropriate credit to the original author(s) and the source, provide a link to the Creative Commons license and indicate if changes were made.

The images or other third party material in this chapter are included in the chapter's Creative Commons license, unless indicated otherwise in a credit line to the material. If material is not included in the chapter's Creative Commons license and your intended use is not permitted by statutory regulation or exceeds the permitted use, you will need to obtain permission directly from the copyright holder.

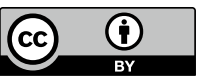

